\title{
Crisis de acceso a la vivienda y mecanismos de promoción del arriendo en Chile: tendencias y desafíos para la consolidación de una Política de Arriendo
}

\author{
Housing crisis and renting in Chile: trends and \\ challenges for the development and consolidation of \\ a Rental Housing Policy
}

Crise no acesso à habitação e mecanismos de promoção ao aluguel no Chile: tendências e desafios para a consolidação de uma Politica do Aluguel

Francisca Bogolasky Fliman

Socióloga, Pontificia Universidad Católica de Chile; Magíster en Administración Pública, Columbia University; PhD en Políticas Públicas, University of Texas at Austin

Postdoctoral Research Fellow, LBJ School of Public Affairs, University of Texas at Austin, Austin, Estados Unidos

fbogolasky@utexas.edu

http://orcid.org/0000-0003-4470-2752

\section{Rosario López Davidson}

Socióloga y Planificadora Urbana, Magíster en Desarrollo Urbano, Pontificia Universidad Católica de Chile Investigadora, Fundación Alcanzable, Santiago, Chile

rlopez3@uc.cl

http://orcid.org/0000-0003-32687583

\section{María Pía Mora Camus}

Socióloga, Pontificia Universidad Católica de Chile

Directora Ejecutiva, Fundación Alcanzable, Santiago, Chile

piamora@alcanzable.cl

http://orcid.org/0000-0002-5051-3248

\begin{abstract}
Resumen
La politica habitacional chilena durante los últimos 50 años ha promovido la tenencia en propiedad a través de la entrega de subsidios a la demanda, lo que, en gran medida, ha consolidado un pais de propietarios. Pese a que durante algunas décadas se redujo el déficit habitacional, los resultados en términos cualitativos han sido deficientes y el aumento reciente del déficit habitacional sugiere que la política habitacional no puede descansar únicamente en este mecanismo. Así, durante la última década también se han integrado otros instrumentos para asegurar el acceso a la vivienda, como lo son los subsidios de arriendo a la demanda. A través de la revisión de documentos institucionales y académicos, entrevistas con actores claves y análisis de datos administrativos,
\end{abstract}


en este artículo se sistematiza la evidencia existente de los tres principales subsidios de arriendo vigentes en Chile -el programa Subsidio de Arriendo, el Programa Vivienda Primero y los Subsidios de Arriendo Transitorios para residentes de campamentos- como también sus resultados y desafíos. Si bien se valora la implementación de herramientas complementarias a la propiedad, los resultados reflejan importantes problemáticas y desafíos, asociados principalmente a la mantención de un enfoque parcial, restringido a los subsidios a la demanda, que no es efectivo para enfrentar el aumento de los valores del suelo y la vivienda, entre otras barreras identificadas. Finalmente, se plantean algunas propuestas para avanzar en una politica de arrendamiento en Chile.

Palabras clave: Arriendo, subsidio de arriendo, política habitacional, vivienda, asequibilidad de la vivienda, precio de la vivienda.

\begin{abstract}
In the last decades, housing policy in Chile has promoted homeownership through subsidies provided to low- and middle-income households to buy homes produced by the private sector. While for several years the housing deficit decreased, there is extensive evidence of qualitative problems, such as the quality and location of the housing units. Additionally, the past years have seen an increase in housing deficit, going back to the 1990s levels, which suggests that housing policy cannot only rely on these types of mechanisms to solve the housing crisis. In the past decade, policy has aimed to address these issues through different mechanisms, including the introduction of rental housing subsidies. Through the analysis of academic and institutional documents and evidence, interviews with key actors, and analysis of administrative data, this article systematizes part of the existing evidence and challenges on three of the main rental subsidies currently in place: the Rental Voucher, Housing First Program and Temporary Subsides for residents of informal settlements. Despite the relevance of these programs to address the housing challenges of some specific populations, the results suggest some important barriers and challenges that need to be considered. These are associated mainly to the sole focus on demand side subsidies, which is not enough to confront the increasing land and housing prices, among other barriers. The article concludes with some proposals to advance in the formulation of a rental housing policy in Chile.
\end{abstract}

Keyword: Renting, renting vouchers, housing policy, housing, housing affordability, housing prices.

\title{
Resumo
}

A política habitacional chilena nos últimos 50 anos tem promovido a propriedade imobiliária por meio da concessão de subsídios sob demanda, o que, em grande medida, consolidou um pais de proprietários. Apesar de durante algumas décadas ter ocorrido redução do déficit habitacional, os resultados em termos qualitativos têm sido ruins e o aumento recente do déficit habitacional sugere que a politica habitacional não pode contar apenas com este mecanismo. Assim, na última década, outros instrumentos também foram integrados para garantir o acesso à moradia, como o subsidio para aluguel à vista. Por meio da revisão de documentos institucionais e acadêmicos, entrevistas com os principais interessados e análise de dados administrativos, este artigo sistematiza as evidências existentes dos três principais subsidios de aluguel em vigor no Chile - o Programa Subsidio de Arriendo, o Primeiro Aluguel de Habitação e Temporário Subsídios para residentes do acampamento - bem como seus resultados e desafios. Embora se valorize a implementação de ferramentas complementares de propriedade, os resultados refletem problemas e desafios importantes, principalmente associados à manutenção de uma abordagem parcial, restrita a subsídios à procura, que não é eficaz para fazer face ao aumento dos valores. Terrenos e habitação, entre outros barreiras identificadas. Finalmente, algumas propostas são feitas para fazer avançar uma política de arrendamento no Chile.

Palavras chave: Aluguel, aluguel social, politica habitacional, déficit habitacional, acessibilidade de habitação. 


\section{Descripción del Problema Público: La política habitacional en Chile, crisis de acceso a la vivienda y la introducción del subsidio de arriendo}

La política habitacional promovida desde el Estado en las últimas décadas ha descansado principalmente en la entrega de vivienda en propiedad, mediante la generación de subsidios a la demanda. El supuesto es que, aumentando la capacidad de pago de los hogares mediante un aporte público, éstos serán capaces de ingresar y competir en el mercado regular de vivienda. En ese sentido, el Estado ha adoptado un rol principalmente subsidiario -consignado constitucionalmente-, entregando por contrapartida un papel protagónico al sector privado en la provisión de soluciones habitacionales, en el marco de un mercado de suelo desregulado.

Los apoyos públicos se han focalizado en los hogares de menores ingresos, mediante la entrega de subsidios que cubren prácticamente la totalidad del valor de la vivienda, sin que deban asumir endeudamiento. También existe cobertura de grupos medios, abarcando hasta el $70 \%$ de la población ${ }^{1}$. A medida que decrece la vulnerabilidad, disminuyen también los aportes que realiza el Estado, debiendo los hogares de ingresos medios complementar los subsidios públicos con ahorro y/o crédito bancario.

Esta aproximación de la política habitacional tuvo logros cuantitativos, siendo uno de los principales la reducción del déficit habitacional heredado de la dictadura; se estima que entre los años 1992 y 2002 se construyeron cerca de 1.270.000 viviendas (CáceresSeguel, 2017). Asimismo, la política de vivienda ha incidido en la consolidación de una gran proporción de propietarios a nivel nacional, sobre todo en los segmentos de menores ingresos. Esto contrasta con lo observado en otros países, tal como USA, Francia y otros, donde a menor nivel de ingresos, mayor es la presencia de arrendatarios (Blanco et al., 2014). El acceso a la vivienda mediado por la acción estatal ha contribuido también a una elevada formalidad de las soluciones habitacionales existentes.

Pese a estos avances, la literatura también ha sido profusa en denunciar las deficiencias asociadas a la forma bajo la cual se produjo la vivienda en Chile en las últimas décadas, siendo la baja calidad, alejada localización y la falta de integración funcional y social algunas de las más subrayadas. Producto de la liberalización del mercado del suelo, y de un enfoque de reducción del déficit cuantitativo, se generó el desplazamiento de la población vulnerable a la periferia y, con ello, la consolidación de extensos conjuntos homogéneos de vivienda social de gran densidad poblacional en los márgenes de la ciudad (Ducci, 1997, 2008). Así, la concentración de la precariedad y la construcción de viviendas de bajo estándar constructivo y alejadas de los bienes públicos urbanos acentuaron e incrementaron los problemas asociados a la vulnerabilidad social (Hidalgo, Paulsen y Santana, 2016; Ducci, 1997; Gledhill, 2010).

A partir de la década del 2000, se han creado diferentes instrumentos para intentar corregir los problemas asociados al stock existente, y otros se han introducido con el fin de prevenir la generación de nuevas situaciones de segregación socio residencial. Éstos abarcan desde regulaciones más exigentes sobre la calidad de la vivienda y sobre la provisión de equipamiento comunitario a nivel barrial, hasta inversión pública para la recuperación de conjuntos habitacionales deteriorados y para la generación de nueva infraestructura pública. También destaca la creación de incentivos a desarrolladores privados para la construcción de proyectos inmobiliarios que alberguen residentes de ingresos mixtos (los denominados proyectos de integración social, regulados por el DS $\left.N^{\circ} 19\right)$.

En este contexto, surge en 2014 el Programa Subsidio de Arriendo, que viene a tensionar la tradición de propiedad, arraigada no sólo en nuestra política pública, sino también a nivel cultural. Este instrumento consiste en un aporte económico estatal a los hogares para pagar un arriendo en el mercado privado, inspirado por el Housing Choice Voucher Program de Estados Unidos. Sin embargo, los subsidios han tenido una muy baja conversión, lo que se da en un contexto de recrudecimiento del déficit habitacional: la encuesta CASEN 2017 reportó un déficit cercano a 500.000 unidades, quebrando así la tendencia a la baja que se observaba en las últimas décadas. A esto se suman los resultados del catastro nacional de cam-

1 La clasificación socioeconómica de los hogares se realiza principalmente mediante la aplicación de un instrumento denominado "Registro Social de Hogares", que utiliza como base datos administrativos y también información declarada por las propias familias. 
pamentos 2020 (TECHO-Chile y Fundación Vivienda, 2021) que revelaron un incremento del $74 \%$ de las familias viviendo en campamentos, con respecto al 2019, afectando a 81.643 hogares.

Tras realizar una breve contextualización sobre el arriendo en Chile, este artículo busca sistematizar la información existente respecto a los instrumentos vigentes de arriendo en el país. Mediante la revisión de documentos institucionales y académicos, entrevistas con actores claves, y análisis de datos administrativos, el artículo realiza un análisis descriptivo sobre el Programa Subsidio de Arriendo, el programa Vivienda con Apoyo y el programa de Arriendo para Residentes de Campamentos, sintetizando sus principales características, y algunos resultados. Además, describe algunas hipótesis para intentar explicar las barreras asociadas a estos programas, tal como la exigua conversión del Subsidio de Arriendo, especialmente en el contexto de ciudades de mayor tamaño, como Santiago. Algunas de estas barreras se relacionan con atributos específicos de los instrumentos, pero otras aluden a aspectos más generales, vinculados al diseño de la política habitacional en general, y los supuestos bajo la que ésta opera. Finalmente, se realizan algunas propuestas para intentar avanzar en una política de arrendamiento en Chile.

\section{El arriendo en Chile: consolidación de la tendencia y elevados precios}

Como se introdujo anteriormente, Chile es mayoritariamente un país de propietarios, con un $62 \%$ de propietarios a nivel nacional, según la última encuesta CASEN. La misma encuesta muestra, sin embargo, que desde el año 2006 se observa un aumento sostenido de la tenencia en arriendo, pasando de un $16 \%$ a un $23 \%$ el año 2020. La proporción de arrendatarios varía según variables territoriales -siendo más predominante a mayor tamaño de la ciudad-, y socioeconómicas -mayor ingreso a mayor arriendo-. Comparado al 2017, llama la atención que en 2020 se haya registrado un incremento de 2,5 y 2,6 puntos porcentuales de esta forma de tenencia para los dos primeros quintiles de ingreso, mientras que para el quintil más alto se registró una disminución de 1,8 puntos porcentuales.
Las razones tras la consolidación de la renta en Chile son diversas, y se asocian a una mayor presión desde la demanda -por ejemplo, cambios en preferencias residenciales y reducción del tamaño de los hogares - como también a barreras para acceder a la vivienda en propiedad -aumento de precios, (que han aumentado más que los ingresos), y también las condiciones establecidas por bancos para el otorgamiento de crédito (tales como tasas de interés, plazos de los créditos y exigencia de ahorro). A eso se suman otros factores estructurales, como la precarización del mercado del trabajo, que vuelve difícil el contar con ingresos formales y estables para un número relevante de familias (Toctoc.com, 2020).

Aunque el crecimiento de los precios de arriendo ha experimentado un crecimiento más controlado que los de venta (Simian, 2018, Toctoc.com, 2020), la carga económica que significa para los hogares sigue siendo desproporcionada. Por ejemplo, para la RM, se estima que el $57,2 \%$ de los hogares correspondientes al 40\% más vulnerable enfrentan un gasto en arriendo que supera el 30\% de sus ingresos. Esto se aleja del estándar esperado, que establece que el gasto en vivienda debiera representar el $25 \%$ o máximo el $30 \%$ del presupuesto de un hogar (Centro de Políticas Públicas UC, 2018, 2020).

\section{Descripción de la lntervención: Mecanismos de Arriendo Vigentes}

Son tres los principales instrumentos implementados en Chile para abordar el arriendo: el Subsidio de Arriendo, el Programa Vivienda con Apoyo, y el Subsidio destinado a Residentes de Campamentos. A continuación, se presenta una síntesis de sus principales características y resultados.

\section{Subsidio de Arriendo (DS N52)}

El Subsidio de Arriendo, regulado por el Decreto Supremo $\mathrm{N}^{\circ} 52$, es actualmente el programa más masivo de apoyo al arriendo. Desde el 2014, el programa ha beneficiado al menos a 26 mil hogares de ingresos bajos y medios, mediante un aporte mensual para arriendo. El programa opera a través de un llamado regular (para jefes de hogar entre 18 y 59 años) y un llamado para adultos mayores (sobre 60 años) ${ }^{2}$.

\footnotetext{
2 Adicionalmente, se han realizado dos llamados orientados a la clase media, uno de los cuales en contexto de pandemia por Covid-19. Debido a su carácter excepcional, este artículo no profundizará en ellos.
} 
El objetivo declarado del programa ha sido entregar un apoyo que responda a las necesidades de movilidad social, laboral y habitacional de familias allegadas o que actualmente ya pagan un arriendo, y se enfoca en hogares de hasta el 70\% más vulnerable según el Registro Social de Hogares ${ }^{3}$. Además, se exigen ciertos requisitos de postulación y activación tales como criterios de edad, tamaño del hogar (mínimo dos personas para el llamado regular y una persona para el llamado de adultos mayores), así como contar con 4 UF de ahorro (para llamado regular), y acreditar ingresos entre 5 y 25 UF (pensión asistencial vigente para adultos mayores $)^{4}$. Por otra parte, no pueden postular, entre otros, personas que ya posean otro subsidio, propietarios de vivienda o postulantes al DS $\mathrm{N}^{\circ} 49^{5}$.

El llamado regular entrega un aporte máximo de 4,2 UF mensuales por concepto de arriendo (4,9 UF en algunas zonas geográficas específicas tal como la RM), que pueden usarse en viviendas con arriendos de hasta 11 UF (13 UF en las zonas geográficas especiales). El subsidio entrega un aporte temporal por un máximo de ocho años, pero en la práctica, si es que se utiliza de manera continua, tiene una duración máxima menor a tres años (cada beneficiario recibe una bolsa de 170 UF, y el subsidio caduca una vez que este monto se utiliza completamente). Una vez seleccionados, los beneficiarios tienen 24 meses para activar su contrato de arriendo. ${ }^{6}$

A la fecha, han postulado cerca de 100 mil personas, siendo seleccionadas cerca del 70\% de ellas. Si bien el programa se ha ido consolidando a través del tiempo, con la ampliación de la población objetivo y aumento de los recursos destinados, una de sus mayores complejidades se relaciona a su baja tasa de aplicación, con aproximadamente $40 \%$ promedio de uso del subsidio a nivel nacional, cifra que disminuye a un 25\% en la RM y aún más en comunas con arriendos más competitivos (se observa una activación algo mayor entre adultos mayores) (MINVU, 2021).
El perfil de los beneficiarios y de quienes aplican el subsidio es similar, siendo una población mayoritariamente femenina, joven, en su mayoría pertenecientes a los grupos más vulnerables, con hogares de 2 o 3 personas, y en su mayoría chilenos, aunque es el programa habitacional con mayor proporción de participantes extranjeros (MINVU, 2021). Sin embargo, y aunque las diferencias no son grandes, se ha observado que quienes logran activar el subsidio suelen vivir en comunas con menor déficit habitacional, menor proporción de arriendo informal y precios de arriendo más bajos, comparado a quienes no utilizan el subsidio (Bogolasky, 2021). A su vez, quienes ya eran arrendatarios activan en mayor medida ( 7 puntos porcentuales más) comparado a quienes eran allegados, considerando que los primeros ya tienen capacidad de pagar un arriendo (López, 2020).

Para quienes usan el subsidio, se observa una tendencia a la proximidad residencial. Así, la mayoría lo utiliza en su región y comuna de origen. Esto se corresponde con las preferencias residenciales de los beneficiarios, donde la mayoría desea quedarse en su misma comuna. En línea con las preferencias, se observa que los atributos residenciales más relevantes para los beneficiarios son la seguridad, tranquilidad, proximidad a redes de apoyo, la accesibilidad del barrio y viviendas de espacio y calidad suficientes (Link et al., 2021; López, 2020).

\section{Programa Vivienda con Apoyo}

El año 2019, y en el contexto de un aumento sostenido de las personas en situación de calle, fue implementado el programa Vivienda con Apoyo del Ministerio de Desarrollo Social. El programa está inspirado en el modelo internacional Housing First, que opera bajo el paradigma que en base a la seguridad de tenencia de la vivienda es posible alcanzar otros objetivos y abordar otras problemáticas que experimenta esta población

3 Instrumento de clasificación de los hogares que permite focalizar apoyos públicos. Se completa en base a datos administrativos y también a información aportada por las personas.

4 Estos son los requisitos actuales, que se han ido modificando y flexibilizando con el tiempo, reflejando la consolidación del programa y su expansión a nuevos grupos poblacionales. Por ejemplo, en 2016 se abrió el programa a todos los hogares con jefes de hogar mayor a 18 años, cuando originalmente estaba dirigido a la población joven de entre 18 y 30 años.

5 Programa de vivienda en propiedad orientado al segmento más vulnerable de la población, que no implica endeudamiento bancario.

6 Actualmente, se encuentran en discusión nuevas modificaciones a los beneficios, incluyendo un aumento de la bolsa de 170 a 210 UF, aumentos en los topes de arriendo hasta 15 UF y ampliación hasta el 80\% de vulnerabilidad, entre otros. 
(salud física, salud mental, estabilidad financiera, recomposición de vínculos sociales, entre otros).

El programa busca resolver la falta de vivienda y dar acceso a servicios de apoyo a personas en situación de calle, y está orientado a personas que llevan al menos cinco años en situación de calle, y que presentan cierto grado de deterioro psicosocial. El funcionamiento es a través de organizaciones sin fines de lucro, que reciben, a través de licitaciones, recursos desde el Ministerio de Desarrollo Social (y más recientemente también desde el Ministerio de Vivienda y Urbanismo), para hacerse cargo de la ejecución del programa. Estas instituciones seleccionan a los postulantes, encuentran y gestionan la estadía en las viviendas, e implementan el trabajo de los equipos multidisciplinarios de intervención (Ministerio de Desarrollo Social, 2021).

El programa define 3 momentos sucesivos que dan estructura al programa. Primero, el momento de difusión y selección de participantes, luego la adaptación y llegada a la vivienda y finalmente el desarrollo del plan personalizado de apoyos (Ministerio de Desarrollo Social, 2021). Los usuarios deben compartir la vivienda con uno o dos participantes más y reciben visitas periódicas por parte del equipo de apoyo. Se busca que este apoyo sea especializado y personalizado, y se basa en cuatro componentes: gestión de la vivienda, integración socio comunitaria, servicios de salud especializados y apoyo domiciliario. Desde su implementación, el programa ha apoyado a cerca de 353 personas, a través de 8 instituciones. Además, el programa tiene una tasa de retención de $86 \%$, y los hallazgos muestran que la mayor parte de los participantes mantienen un vínculo activo con sus redes familiares, con los servicios de salud y la mayoría mejora sustancialmente su bienestar subjetivo.

\section{Arriendo para Residentes de Campamentos}

Finalmente, existen también los subsidios de arriendo transitorios para residentes de campamentos, que se entregan a residentes de asentamientos informales en proceso de radicación o erradicación, en el marco del Programa Asentamientos Precarios del MINVU. Hay evidencia de su utilización desde el 2011, pero se aplica de forma más sistemática desde el año 2017. Los subsidios no tienen duración determinada; se entregan inicialmente por 12 meses, siendo renovados hasta la entrega de las viviendas en propiedad.

La operación y financiamiento de estos subsidios se realiza a través de diferentes mecanismos, los que se distinguen principalmente por los actores involucrados y el monto y origen de los recursos utilizados: los Gastos de Traslado Transitorios (GTT), los Subsidios de Albergue Transitorios (STT), las Asignaciones directas del Subsidio de Arriendo y el Fondo ORASMI. El mecanismo más utilizado es el GTT, donde a través de una solicitud levantada por el Serviu a la División de Política Habitacional del MINVU, se transfieren recursos a Municipalidades, organismos públicos u otras instituciones sin fines de lucro, las que gestionan con las familias el pago del arriendo hasta la entrega de la vivienda definitiva ${ }^{7}$.

Se estima que, con los GTT, desde el año 2017, se ha apoyado a cerca de 262 familias provenientes de 12 campamentos de la RM, con un subsidio mensual promedio de $\$ 232.000$ y con una duración promedio de 18 meses. El valor mensual del subsidio ha aumentado con el pasar de los años, teniendo hoy un tope de $\$ 300.000$. Pese a que el programa permite un copago, esto es inusual, y en general se arriendan viviendas dentro del valor del subsidio.

\section{Análisis: Tendencias, Barreras y Desafíos}

Si bien cada uno de los programas tiene una orientación y un funcionamiento singular, es posible identificar ciertas tendencias, barreras y desafíos comunes.

Dada la considerable demanda por un arriendo protegido, la baja activación de los subsidios es el resultado más problemático de los programas de arriendo, limitando su potencial para dar acceso a una vivienda adecuada. De acuerdo con la evidencia existente, existen diversas barreras por parte de la oferta y la demanda que podrían estar incidiendo en el funcio-

7 Los STT, las asignaciones directas del DS N52 y el FONDO ORASMI tienen una aplicación similar, solo que su uso responde a circunstancias de mayor emergencia y excepcionalidad, siendo los GTT el mecanismo regular para dar solución habitacional transitoria a los residentes de campamentos. 
namiento de los programas y en el uso efectivo de los subsidios (barreras que a su vez dependen del funcionamiento y condiciones de los programas).

En cuanto a la oferta de arriendo, primero, está la asequibilidad de los arriendos. Gran parte de los arriendos vacantes no solo no cumplen con los topes de arriendo del programa, sino también con el presupuesto de copago de las familias. Se estima que durante la última década hubo un alza promedio de un $43 \%$ en los valores reales de los arriendos. Así, según evidencia reciente, solo $12 \%$ de los avisos de arriendo online de departamentos en el Gran Santiago son elegibles en el marco del Subsidio de Arriendo y los de casas serían prácticamente inexistentes (Link et al., 2019, 2021).

La asequibilidad también tiene que ver con la invisibilidad de la oferta y la falta de viviendas que cumplan con las necesidades de los hogares. Por un lado, los arriendos asequibles para estos hogares no aparecen en los portales web tradicionales de vivienda. La mayoría de los beneficiarios acabaría encontrando los arriendos a través de datos de conocidos, búsqueda en terreno y Facebook (Díaz, 2015; MINVU, 2018; Link et al., 2021). Por otro lado, la mayor parte de la oferta asequible no cumple con los estándares básicos de acuerdo con la composición de las familias (por ejemplo, la creciente oferta de departamentos studio, parte importante de la oferta de departamentos asequibles en términos económicos, no es compatible con núcleos con hijos).

Segundo, la poca disposición de los arrendadores para arrendar con los diferentes subsidios de arriendo. Entre las causas identificadas, se observa desconfianza en el pago del arriendo -ya sea hacia el Gobierno o el beneficiario, la percepción de una alta burocracia para participar de los programas como arrendadores, la desinformación sobre los programas y los usuarios -y un consecuente prejuicio y discriminación, la tendencia a la informalidad del arriendo entre los grupos de menores ingresos, y el no cumplimiento de los requisitos económicos requeridos a los hogares, entre otras. Esto además se vería acentuado por algunos requisitos de los programas, tales como la prohibición de arrendar viviendas a parientes y sin recepción municipal (MINVU, 2018; Link et al., 2021; Bogolasky, 2021; López, 2020).

Respecto a los usuarios de los programas, hay evidencia de problemas de desinformación entre la población objetivo y beneficiarios sobre la existencia, características y funcionamiento de los programas. Se reconoce una falta de estrategias para transmitir la información necesaria para postular y aplicar los subsidios, problema aún mayor entre la población adulta mayor (MINVU, 2019). En el caso del Subsidio de Arriendo Regular, por ejemplo, incluso se ha observado que los beneficiarios no se enteran de que han sido beneficiados, no conocen los requisitos de la vivienda a arrendar o no conocen los plazos asociados (Link et al., 2021; MINVU, 2018).

Además, a pesar de contar con un subsidio, el copago significa una gran carga en los ingresos de muchos hogares. Asimismo, los programas no consideran otros gastos de la vivienda, tales como el pago de servicios y gastos comunes, que pueden llegar a representar montos similares a los del copago. Así, por ejemplo, allegados o residentes de campamentos que son beneficiados con un subsidio, desisten de utilizarlo y optan por vivir (o seguir viviendo) bajo la situación de allegamiento.

Con rasgos similares con lo que sucede con la vivienda en propiedad, se identifica que parte del problema de aplicabilidad de los subsidios tiene su origen en que los programas, bajo los principios subsidiarios de la demanda, solo entregan recursos a los usuarios, sin intervenir de manera significativa en la oferta de arriendo disponible y en la promoción de nueva oferta asequible, generando un desajuste entre la oferta y la demanda del arriendo entre los sectores de menores ingresos.

\section{Conclusiones y Recomendaciones: Hacia una Política de Arriendo}

Los tres instrumentos revisados comparten el ser vouchers dirigidos a la demanda, que deben aplicarse en el mercado privado de viviendas. En el contexto internacional, se observa que este tipo de apoyos se da de forma complementaria a otras medidas, como las orientadas a la generación de oferta a precios asequibles, entre otros (Centro de Políticas Públicas UC, 2018). Si bien en Chile hay ejemplos interesantes de generación de oferta de arriendo asequible, como es el caso del proyecto Justicia Social I, liderado por la Corporación Municipal Innova Recoleta, estos aún son incipientes y aislados.

La consolidación de un sistema de arriendo protegido -y, en términos más amplios, de una política habitacional exitosa- no ocurrirá de forma espontánea; se 
requiere abordar este desafío con un enfoque integral, que considere al menos los siguientes elementos:

- Gobernanza: hoy existen subsidios de arriendo provistos por entidades del gobierno central, como el Ministerio de Vivienda y el Ministerio de Desarrollo Social, siendo la articulación con otros sectores (como el Ministerio de Transportes) y otros niveles del Estado (como los municipios) prácticamente inexistente. Tampoco se ha innovado mayormente en la incorporación del sector privado sin fines de lucro, como ocurre a nivel internacional, donde las Asociaciones de Vivienda juegan un rol central en relación a la creación de stock de arriendo y su administración. Un reto adicional lo constituye la promoción de la participación activa de los usuarios; por ejemplo, a través de la conformación de organizaciones como las Federaciones de Inquilinos, que se involucren en la defensa de sus derechos y en la toma de decisiones.

- Regulación: La planificación urbana debe ser el marco dentro del cual se inscriben los instrumentos de arriendo. Sin una adecuada regulación del suelo, que favorezca la generación de nuevas viviendas alcanzables, así como también que incentive la regeneración urbana con criterios de integración social, la aplicación de los subsidios a la demanda difícilmente llegará a buen término.

- Financiamiento: de forma complementaria a los vouchers a la demanda, se requiere de nuevos instrumentos financieros que incentiven directamente la generación de oferta, como por ejemplo la entrega de préstamos bajo condiciones preferentes, y que el Estado asuma un rol de garante ante créditos solicitados ante la banca privada. Otras alternativas son la entrega de suelo público y la generación de exenciones tributarias para quienes desarrollan proyectos de arriendo social.

- Articulación entre programas de vivienda de arriendo y propiedad: hoy, los programas de propiedad no se articulan adecuadamente con los de arriendo, lo que puede dificultar la amplificación de estos últimos. Por ejemplo, en los proyectos de vivienda de integración social (DS $N^{\circ} 19$ ), no se contempla regularmente la incorporación de unidades habitacionales orientadas al alquiler. Lo mismo vale para los programas DS $\mathrm{N}^{\circ} 49$ o DS
$\mathrm{N}^{\circ} 1$. Por otro lado, los incentivos a pasar por el arriendo previo acceso a la propiedad son aún bastante incipientes.

Sólo adoptando estas medidas será factible tanto responder a la demanda insatisfecha por alquiler de carácter social existente hoy en Chile, como también incrementar el interés en esta forma de tenencia.

\section{Referencias}

BLANCO, A., FRETES, V. Y MUÑOZ, A. (2014). Busco casa en arriendo. Promover el alquiler tiene sentido. Washington D.C.: Banco Interamericano de Desarrollo. https://politicaspublicas.uc.cl/wp-content// uploads/2015/06/FMM_Book_Busco_casa_en_arriendo.pdf

BOGOLASKY, F. (2021). The Paradox: When poor housing conditions act as a barrier to better housing. [Tesis doctoral, University of Texas at Austin]. Austin, TX.

CÁCERES-SEGUEL, C. (2017). Vivienda social periurbana en Santiago de Chile: la exclusión a escala regional del trasurbanita de Santiago de Chile. Economía, sociedad y territorio, 17(53), 171-198. https://doi. org/10.22136/est002017664

CENTRO DE POLÍTICAS PÚBLICAS UC. (2018). Estudio comparado de experiencias internacionales sobre la administración de bienes inmuebles para arriendo. Subdirección de Investigación Aplicada Centro de Políticas Públicas UC. https://politicaspublicas.uc.cl/ wp-content//uploads/2018/12/20180807_-Informe-2_ casos-internacionales.pdf

CENTRO DE POLÍTICAS PÚBLICAS UC. (2019). Administración de Arriendo Asequible: Evidencia Internacional y Propuestas para Chile. Seminario Centro de Políticas Públicas UC, Santiago, Chille. https:// politicaspublicas.uc.cl/wp-content//uploads/2018/12/ PPT-Seminario-Arriendo.pdf

CENTRO DE POLÍTICAS PÚBLICAS UC. (2020). Hacia la consolidación de una política de arriendo en Chile. Evaluación económica de un modelo de provisión de vivienda asequible sobre suelo público, mediante sociedades mixtas. Seminario Centro de Políticas Públicas UC, Santiago, Chile. https://politicaspublicas.uc.cl/ wp-content//uploads/2020/12/Presentacio\%CC\%81nmodelo-de-arriendo-asequible.pdf

DÍAZ, C. (2015). "Chao Suegra": El subsidio de arriendo y los efectos en la movilidad y localización residencial de sus beneficiados en el Sistema Urbano Metropolitano de Santiago (2014-2015) [Tesis de Magister en Desarrollo Urbano, Pontificia Universidad Católica de Chile]. 
DUCCI, M. (1997). Chile: el lado oscuro de una política de vivienda exitosa. Revista EURE, 23(69), 99115. https://repositorio.uc.cl/xmlui/bitstream/handle/11534/3648/000159091.pdf

DUCCI, M. (2008). La política habitacional como instrumento de desintegración social. Efectos de una política de vivienda exitosa. En: C. Barba Solano (Ed.). Retos para la integración social de los pobres en América Latina (pp. 293-310). Buenos Aires: CLACSO.

GLEDHILL, J. (2010). El derecho a una vivienda. Revista de Antropología Social, 19, pp. 103-129. https:// revistas.ucm.es/index.php/RASO/article/view/RASO1010110103A

HIDALGO, R., PAULSEN, A. Y SANTANA, L. (2016). El neoliberalismo subsidiario y la búsqueda de justicia e igualdad en el acceso a la vivienda social: El caso de Santiago de Chile (1970-2015). Andamios, 13(32), 57 81. http://dx.doi.org/10.29092/uacm.v13i32.525

LINK, F., MARÍN TORO, A. Y VALENZUELA, F. (2019). Diagnóstico y propuestas para un sistema integral de vivienda en arriendo con interés social. Temas de la Agenda Pública, $\mathrm{N}^{\circ}$ 114. https://politicaspublicas.uc.cl/wp-content//uploads/2019/07/ Diagn\%C3\%B3stico-y-propuestas-para-un-sistemaintegral-de-arriendo.pdf

LINK, F., MARÍN, A., VALENZUELA, F., CATÁN, C., LÓPEZ, R. Y DÍAZ, J.I. (2021). Tenencia en arriendo en la política habitacional en Chile: Desafío de integración social y urbana. Instituto de Estudios Urbanos y Territoriales UC, Documentos de Trabajo del IEUT, N ${ }^{\circ}$ 19. https://estudiosurbanos.uc.cl/documento/tenencia-en-arriendo-en-la-politica-habitacional-en-chiledesafios-de-integracion-social-y-urbana/

LÓPEZ, R. (2020). Arriendo y movilidad residencial: Preferencias residenciales y resultados de localización de los beneficiarios del Subsidio de Arriendo en Santiago de Chile [Tesis de Magister en Desarrollo Urbano, Pontificia Universidad Católica de Chile].

LÓPEZ. E. Y HERRERA, N. (2018). Arriendos por las nubes: Efecto de la creciente concentración de la propiedad. Ciper Chile. https://www.ciperchile. cl/2018/07/25/arriendos-por-las-nubes-efecto-de-lacreciente-concentracion-de-la-propiedad/

MINVU (2018). Encuesta a beneficiarios Subsidio D.S. 52. Sistema de seguimiento de programas MINVU, Comisión de Estudios Habitacionales y Urbanos. http://calidaddevida.colabora.minvu.cl/ doc2016/2016\%20-\%20Informe\%20Encuesta\%20 Beneficiarios\%20del\%20Subsidio\%20D.S.\%20\%20 N\%C2\%B052.pdf

MINVU (2019). Evaluación focalizada del ámbito de diseño Programa Subsidio de Arriendo. Evaluación para la Dirección de Presupuestos. https://www.dipres.gob. cl/597/articles-189331_informe_final.pdf

MINVU (2021). Caracterización Beneficiarios Subsidio de Arriendo (DS52). Estadísticas Habitacionales Observatorio Urbano. https://www.observatoriourbano.cl/ estadisticas-habitacionales/

MINISTERIO DE DESARROLLO SOCIAL (2021). Manual metodológico Vivienda con Apoyo para personas en situación de calle. Oficina Nacional de Calle, Subsecretaría de Servicios Sociales. https://drive. google.com/file/d/laLvbK5ApcRguoWf7cgC3bivVkb 5150PT/view

SIMIAN, J. (2018). El crecimiento del Mercado de arriendo en Chile. Centro de Estudios Inmobiliarios ESE Business School: Universidad de los Andes. https://www.ese.cl/ese/site/artic/20180927/asocfile/20180927162505/2018_09_mercado_arriendo_habitacional_en_chile_ese_public.pdf

TECHO-CHILE \& FUNDACIÓN VIVIENDA. (2021) Catastro Nacional de Campamentos 2020-2021. Disponible en https://ceschile.org/catastro/

TOCTOC.COM (2020). Una radiografía al mercado del arriendo en el Gran Santiago. Reporte $\mathrm{N}^{\circ} 4$ Apuntes de Ciudad. Área de Estudios Toctoc.com. https://blog.toctoc.com/wp-content/uploads/2021/09/4-Apunte-deCiudad-Arriendo-en-Gran-Santiago.pdf 